\title{
Cause of death in under 5 children in a demographic surveillance site in Pakistan
}

\author{
Muhammad Imran Nisar*, Muhammad Ilyas, Komal Naeem, Urooj Fatima and \\ Fyezah Jehan
}

Pediatrics, Aga Khan University, Karachi, Pakistan

\section{Objective}

To identify Cause of deaths among children below age of 5years from a prospective cohort of women in one urban and four peri-urban settings of Karachi, Pakistan

\section{Introduction}

Pakistan ranks 26th in Childhood mortality rates, globally. Pakistan, with other 4 countries is responsible for about half of the deaths of children age under 5 . Despite such burden vital registration system is not well established, health facilities are not easily accessible and mostly deaths occur at home, making identification of cause of death (COD) difficult

\section{Methods}

From Jan 2007-Dec 2012 under-5 mortality was identified by CHWs during their 3-monthly visits. A Research Assistant conducted Verbal Autopsies (VA). Each VA form was analyzed by 2 physicians, independently, and assigned a cause. VA is analyzed by a third physician in case two physicians do not agree on a cause. Cause Specific Mortality Fractions (CSMF) were calculated for each identified COD.

\section{Results}

833(58\%) neonatal deaths and 591(42\%) Under-5 deaths (excluding neonates) were identified. Among neonates most common CODs were perinatal asphyxia(30.4\%), neonatal sepsis/meningitis(28\%), pre-term birth complication(11\%) and neonatal pneumonia(6\%). For Post-neonatal deaths most common CODs were sepsis (19\%), diarrheal disease (17\%), Pneumonia (17\%) and meningitis (8\%).

\section{Conclusions}

We describe the CSMF for different CODs among neonated and children under 5. Strategies for prevention of most common causes and making health facilities easily accessible will decrease this burden.

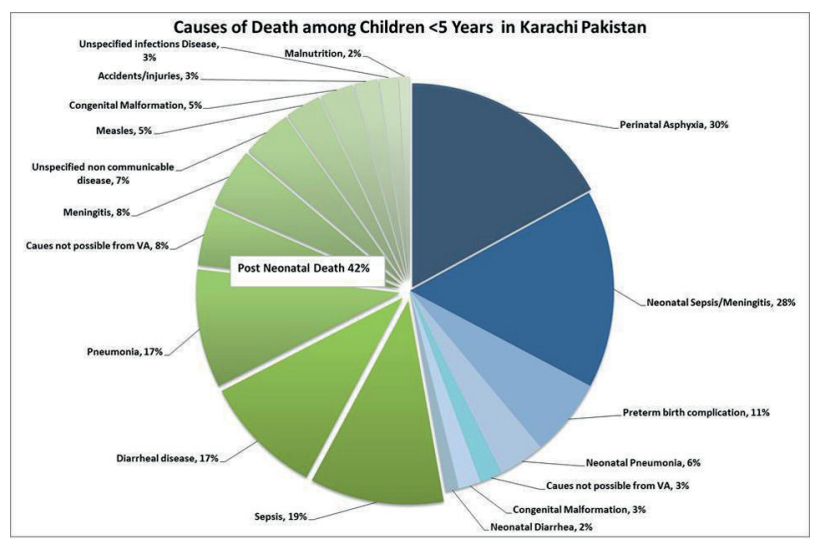

\section{Acknowledgments}

Aga Khan University

*Muhammad Imran Nisar

E-mail: imran.nisar@aku.edu

Keywords

Mortality; children; neonates 